\title{
APLICACIÓN DE UNA ESTRATEGIA METDOLOGICA PARA EL APRENDIZAJE DEL CONCEPTO FRUTO
}

\author{
Eblis Álvarez S., Myriam Clavijo \\ Profesores Universidad Pedagógica Nacional
}

\begin{abstract}
The teaching in botany is mainly related to the transmissionism tendency, for that reason we designed a strategy for learning about the fruit concept.

In this strategy, the student produce and search information from the object and they work in peer colaboration.

Two group of students participated in this study to whom were given oral fundamental understanding about cognitive events. Data about their conceptions were collected using sorne questions about the fruit concept. After, a pretest was employed to examine cognitive aspectos about the fruit, before workshop was made and then was applied like postest, finally they solve sorne problems. The results about their conceptions indica te ceriain coincidence in their notions.
\end{abstract}

Pretest and postest results indica te a low conceptual progress in both groups and the to solve problems capacity was low too.

Palabras claves: aprendizaje, estrategia, fruto.

\section{RESUMEN}

La enseñanza de la botánica se ha llevado a cabo bajo la tendencia transmisionista, por ello se diseñó una estrategia para el aprendizaje del concepto fruto.

En dicha estrategia el estudiante produce y busca información del objeto de estudio y realiza un trabajo de grupo.

Des grupos de estudiantes participaron en este estudio a quienes inicialmente se les suministró en forma oral algunas ideas acerca de posibilidades en el desarrollo cognitivo si hay un compromiso para ello.

En el trabajo se diagnosticaron preconcepciones acerca del concepto fruto y luego se aplicó un pretest, para indagar aspectos cognitivos del concepto, posteriormente se desarrolló un taller y se aplicó de nuevo el pretest. Finalmente se aplicaron algunos problemas para su resolución.

Los resultados muestran un bajo dominio conceptual al igual que una baja capacidad en la resolución de problemas. 


\section{INTRODUCCIÓN}

La experiencia como docentes en el área de botánica nos ha llevado a repensar la forma metodológica de abordar el trabajo procedimental y conceptual en dicha área, lo cual nos llevó a desarrollar una línea de trabajo sobre el aprendizaje de algunos conceptos fundamentales en Botánica — una investigación aula-.

La tendencia transmisionista en la enseñanza de la botánica presenta conceptos acabados lo que dificulta desarrollar la actividad participativa del estudiante permitiendo que él se involucre en su proceso de aprendizaje (Vasco y Aragón, 1996).

Entonces se hace necesario repensar la relación profesor-alumno, en la cual el estudiante pasaría de ser un receptor pasivo a un sujeto activo en gran parte responsable y cor'nprometido en su proceso de aprendizaje y el profesor realizaría el rol de orientador de dicho aprendizaje (Gil, 1991).

El estudiante puede producir información al estar en contacto con los objetos de estudio, en este caso los vegetales, dicha información puede ser ampliada y corroborada con revisiones bibliográficas (Alvarez y Arias, 1996). Mediante el planteamiento de preguntas que guían el trabajo, el profesor induce a los estudiantes a generar procesos mentales para que interrelacionen sus observaciones y lecturas, buscando que cada sujeto llegue a una respuesta parcial, la socialización, discusión y confrontación con lo aceptado por la comunidad de botánicos de dichos planteamientos permitirán llegar en consenso a una respuesta parcial que ha sido elaborada por y para el grupo.

Esta forma de trabajo implica un mayor compromiso y dedicación por parte de estudiantes y profesores para lograr del proceso de apropiación del conocimiento un máximo aprovechamiento, no sólo desde el aspecto de apropiación de desarrollos conceptuales en botánica sino además de desarrollos personales tanto en habilidades como en personalidad tales como capacidad de observación detallada, atención, autonomía, comprensión de lectura, responsabilidad, etc.

Tomando como referencia lo anterior en el presente trabajo se desarrolla una estrategia para el aprendizaje del concepto fruto.

\section{METODOLOGÍA}

La estrategia de trabaja se aplica a dos grupos de 18 y 21 estudiantes del VI semestre de la licenciatura en Biología de la Universidad Pedagógica Nacional durante dos semestres consecutivos.

Para iniciar el presente trabajo se realizó una charla de motivación con la cual se pretendió llegar a los estudiantes para llamar su atención sobre las posibilidades de desarrollos cagnitivos y personales que se podrían lograr si ellos se involucran y comprometen con su propio proceso de aprendizaje, se planteó que el conocimiento científico no es algo acabado y que los desarrollos propios deben contrastarse con lo aceptado por la comunidad botánica para lograr un mismo canal de comunicación, resaltando que lo importante son los procesos que cada individuo desarrolle para lograr su respuesta parcial; la pregunta y las actividades generadas para abordarla serán muy importantes dentro de esta labor. 
Con el fin de establecer las relaciones existentes entre los estudiantes y los objetos de estudio en este caso las trufas, se diseñaron unas preguntas que permitieron establecer las ideas en relación con los frutos adquiridos de la cotidianidad y la historia de vida de cada estudiante.

Para orientar el desarrollo del trabaja tanto desde el aspecto fisiológico como morfológico relacionado con los frutos, se diseñaron y aplicaron talleres que contemplan una parte práctica de manipulación, observación y descripción del material de estudio, una revisión teórica para contrastar bibliográficamente la información producida por el estudiante y una posterior socialización, discusión, y consenso de saberes.

Antes y después del desarrollo de los talleres se aplicó un instrumento de selección múltiple con el cual se pretendió establecer si los estudiantes lograron apropiación del conocimiento trabajado.

Con el propósito de evidenciar la capacidad de interrelación de análisis, y de inferencia lograda por el estudiante, se empleó la estrategia de resolución de problemas relacionados con los objetos de estudio.

\section{RESULTADOS Y DISCUSIÓN}

El trabajo realizado mostró los siguientes resultados:

Al examinar el diagnóstico sobre preconcepciones, en ambas grupos se revela cierta coincidencia en sus ideas, ya que la mayoría establece una relación del fruto con la flor y la semilla, al igual, que su papel en la dispersión de semillas, pero muy pocos lo relacionan can el ovario.

Hay que anotar que muy pocos estudiantes en ambas grupas, establecen una diferencia entre fruto y fruta y en uno de los grupos algunos de los estudiantes consideran algunos tallos y raíces coma frutos.

Con respecto a indagar preconcepciones Sebastia J. (1989), considera que es muy importante conocer las ideas que el estudiante posee acerca de un concepto antes del proceso de enseñanza, además de su utilidad para diseñar estrategias que orientan el trabajo, esta apreciación concuerda con lo expuesta por Mateo (1993).

En lo referente a los resultados de la prueba de selección múltiple, al comparar valares porcentuales del pre-test y post-tes para ambos grupos se detecta que el grado de avance en el manejo conceptual fue muy bajo, muy probablemente porque el compromiso de los estudiantes con la estrategia fue deficiente, especialmente en cuanto a la actitud de producción y búsqueda de información, lo que conlleva a una baja participación y por lo tanto a disminuir la actividad de socialización de saberes, situación que según Arca et al (1990) es muy importante para trabajar y discutir a partir de objetos, permitiendo iniciar un diálogo de sabe res. Ríos y Tibatá (1998) destacan en su trabajo la baja participación de los estudiantes durante la fase interactiva y cuando lo hacían era muy pobre la calidad de las intervenciones, inclinándose más bien hacia las ideas del profesor sin un análisis crítico. Lo cual coincide con lo observado a través de este trabajo.

Hay que hacer notar que en ambos grupos en tres de las preguntas se manifiesta un cierto nivel de desaprendizaje. 
Esto podría deberse a que algunos estudiantes responden las pruebas de selección múltiple al azar ó a que no se logra cambio conceptual.

En las resultadas obtenidas en la resolución de problemas, sólo una minaría de estudiantes en ambos grupos logró resolverlos, ya que en una escala de 0-50 puntos, sólo una pareja logró un valor de 40 puntos, ella puede interpretarse como una baja capacidad de aplicación y extrapolación conceptual, al respecto Gil (1988) comenta que a través de la resolución es como mejor se aprende, porque se obliga a los estudiantes a poner sus conocimientos en práctica y favorecer la comunicación. En tanto que López y Casta (1996) resaltan una cierta ausencia de investigación en este aspecto en el Contexto del Aula.

Hay que destacar que el trabajo se realizó en forma grupal tanto en el aspecto práctico como teórico y la información fue socializada y negociada, a pesar de ello los resultados no fueron los esperados, la cual es contradictoria con lo obtenido por Lampe y Starver (1995) quienes en su artículo plantean que el trabajo en grupa permite un mayor aprendizaje conceptual, la cual es corroborada por Damon y Pheips (1989), quienes consideran que el trabajo en grupo genera un compromiso para aprendizaje mutuo, retroalimentación recíproca y el compartir ideas, aunque estas interacciones depende de la composición y dinámica del grupo.

En general los resultadas obtenidas muestran que la estrategia de trabajo no generó el aprendizaje esperado, probablemente, según una entrevista oral, realizada en una actividad plenaria, porque la forma de trabajo era nueva para ella y no habían tenido la oportunidad de reflexionar, analizar y debatir el conocimiento que trabajan; y parece ser que se les dificulta superar la metodología tradicional o sea la labor centrada en el maestro y son muy reticentes a la participación activa en la producción y búsqueda de información y a que sea el estudiante el Centro del proceso.

\section{BIBLIOGRAFÍA}

Álvarez, E. y Arias, H. -1995. Algunas consideraciones generales sobre el aprendizaje conceptual en botánica. Estudios en pedagogía y didáctica. Vo. $1 \mathrm{~N}^{0} 1$, Abril.

Arca, M. et al, 1990. Enseñar ciencia. Cómo empezar. Cómo empezar: Reflexiones para Educación Científica de base. 1 . Edición. Paidos. Barcelona.

Damon, W. and Phelps, E. 19899. Critical distincions among three fo peer Education International Journal of Educational Research.

Gil, Pérez, D. 1988. La resolución de problemas de lápiz y papel como actividad de investigación. Investigación en la Escuela, $\mathrm{N}^{0} 6$.

Gil Pérez, D. 1991. ¿Qué han de saber y saber hacer los profesores de Ciencias? Enseñanza de las Ciencias. Vol. 9. $\mathrm{N}^{0} 1$.

López y Costa, R. 1996. Modelo de enseñanza aprendizaje centrado en la resolución de problemas. Enseñanza de las Ciencias. Investigación y experiencias didácticas. Vol. 14, $\mathrm{N}^{0}$ 1. Marzo 
Lumpe, A., y Straver J. 1995. Journal of Research in Science teaching: Peer Colaboration in concepto development: Learning about photosinthesis. Vol. 32. $\mathrm{N}^{0} 1$.

Mateos, A. 1993. Ideas previas en botánica. Enseñanza de las Ciencias. Vol. 11, N02.

Ríos, R. y Tibatá, A. 1998. Diseño, Aplicación y Evaluación de una estrategia para orientar la construcción integral del concepto propagación natural en estudiantes de VI semestre de Licenciatura en Biología de la Universidad Pedagógica Nacional. Trabajo de Grado.

Sebastia, J. 1989. El Constructivismo: Un marco teórico - problemático. Enseñanza de las Ciencias. Vol. $7 \mathrm{~N}^{0} 2$.

Vasco, C. y Aragón, M. 1993. Docencia retroactiva y proactiva de la física: Red N ${ }^{0} 3$. 